\title{
Molecules in coorbit spaces and boundedness of operators
}

by

\author{
Karlheinz Gröchenig and Mariusz Piotrowski (Wien)
}

\begin{abstract}
We study the notion of molecules in coorbit spaces. The main result states that if an operator, originally defined on an appropriate space of test functions, maps atoms to molecules, then it can be extended to a bounded operator on coorbit spaces. For time-frequency molecules we recover some boundedness results on modulation spaces, for time-scale molecules we obtain the boundedness on homogeneous Besov spaces.
\end{abstract}

1. Introduction. A remarkable principle of classical analysis states that an operator that maps atoms to molecules is bounded. Here an "atom" is a function on $\mathbb{R}^{d}$ satisfying certain support and moment conditions, and norm bounds. Atoms arose first in the study of atomic decompositions of real Hardy spaces [2] and singular integral operators on Hardy spaces (see $[13,25]$. The notion of an atom was later diversified to adapt to the BesovTriebel-Lizorkin spaces [10], and then generalized to "molecules", which are functions satisfying norm bounds, moment and decay conditions (instead of support conditions); see $[3,10]$. The resulting molecular decompositions of function spaces have been successfully applied to study the boundedness properties of Calderón-Zygmund operators on Besov-Triebel-Lizorkin spaces (see $[8-11,27]$ for some of the main contributions). The technical part of the proofs is to show that the operator under consideration maps smooth atoms into smooth molecules. Using norm estimates for atomic and molecular decompositions, one then obtains the boundedness of the operator. A similar strategy has been used in [14] to study a class of pseudodifferential operators on Besov-Triebel-Lizorkin spaces.

In this paper we study atoms, molecules, and the boundedness of operators in the context of coorbit theory. In coorbit theory one can attach to every irreducible, unitary, integrable representation $\pi$ of a locally compact group $\mathcal{G}$ on a Hilbert space $\mathcal{H}$ a class of $\pi$-invariant Banach spaces Co $Y$ that is parametrized by function spaces $Y$ on the group $\mathcal{G}$. These

2000 Mathematics Subject Classification: 42B35, 46E35.

Key words and phrases: coorbit space, boundedness of operators, representation theory, atom, molecule, modulation space, Besov space, Hilbert transform. 
so-called coorbit spaces possess a rich theory ranging from interpolation properties and duality theory to atomic decompositions and the existence of frames (see the series of papers $[5-7,15]$ ). The best known examples of coorbit spaces are the Besov-Triebel-Lizorkin spaces (by choosing the group of affine transformations on $\mathbb{R}^{d}$ and the representation by translations and dilations) and the modulation spaces (by choosing the Heisenberg group and the Schrödinger representation). An interesting recent example is the family of shearlet spaces of [4].

In the context of general coorbit spaces, the atoms are subsets $\left\{\pi\left(x_{i}\right) g\right.$ : $\left.x_{i} \in \mathcal{G}\right\}$ in the orbit of the representation $\pi$ for suitable $g \in \mathcal{H}$. One of the main results of coorbit theory establishes the existence of atomic decompositions with respect to such atoms $[6,15]$. In the standard examples, these atomic decompositions imply the non-orthogonal wavelet expansions of the homogeneous Besov spaces and the Gabor-type expansions of modulation spaces.

Our contribution is the introduction of molecules in general coorbit spaces and the study of their properties. Roughly speaking, a set of molecules is determined by an envelope function $H$ on the group $\mathcal{G}$ and a discrete subset $\left\{x_{i}\right\}$ of positions in $\mathcal{G}$. See Section 3 for the precise definition. Our main result then shows that any operator that maps a set of atoms $\pi\left(x_{i}\right) g$ to a set of molecules is bounded on the associated coorbit spaces (Theorem 3.5).

We then investigate what the abstract theorem says for the concrete examples of the Schrödinger representation of the Heisenberg group and for the group of affine transformations. For the Heisenberg group we recover the notion of time-frequency molecules which were introduced already in $[1,17,20]$. Our main theorem implies the boundedness of pseudodifferential operators on modulation spaces $[18,20]$. The use of time-frequency molecules sheds a new light on mapping properties of pseudodifferential operators. For the group of affine transformations we investigate explicit time-scale molecules. Our main insight shows that classical smooth molecules are also time-scale molecules in the sense of coorbit theory. As an effortless application of our main result, we verify the boundedness of the Hilbert transform on homogeneous Besov spaces.

The paper is organized as follows. In Section 2 we summarize some of the standard facts of coorbit space theory from $[6,15]$. We recall the necessary definitions of function spaces on locally compact groups and of coorbit spaces, and then describe their atomic decompositions and Banach frames. In Section 3 we introduce the notion of molecules in the context of coorbit spaces and study their fundamental properties. This section contains our main result about the boundedness of operators acting on coorbit spaces: if an operator maps atoms to coorbit molecules, then it can be extended to a 
bounded operator on the corresponding coorbit spaces. Section 4 is devoted to making the abstract theory explicit for the case of the Heisenberg group and of the group of affine transformations.

2. Coorbit space theory. First we recall the concepts and required results from the theory of coorbit spaces. We work with function spaces and representations on a locally compact group.

2.1. Preliminaries and notation. Throughout, $\mathcal{G}$ will be a locally compact group with identity $e$. Integration on $\mathcal{G}$ will always be with respect to the left Haar measure, and $\Delta$ is the the Haar modulus on $\mathcal{G}$. We denote by $L_{x} F(y)=F\left(x^{-1} y\right)$ and $R_{x} F(y)=F(y x), x, y \in \mathcal{G}$, the operators of left and right translation. Further, we also need the involution $F^{\vee}(x)=F\left(x^{-1}\right)$. The space of all bounded functions on $\mathcal{G}$ with compact support will be denoted by $L_{0}^{\infty}(\mathcal{G})$. Let $\chi_{U}$ be the characteristic function of the set $U$.

2.2. Banach function spaces on $\mathcal{G}$. We work in the context of Banach function spaces. We assume that $Y$ is a Banach space consisting of functions on $\mathcal{G}$ equipped with the norm $\|\cdot \mid Y\|$ and that $Y$ has the following properties.

(i) $Y$ is continuously embedded into $L_{\text {loc }}^{1}(\mathcal{G})$, the locally integrable functions on $\mathcal{G}$.

(ii) $Y$ is solid, i.e., if $F \in Y, G$ is measurable and satisfies $|G(x)| \leq$ $|F(x)|$ a.e., then $G \in Y$ and $\|G|Y\|\leq\| F| Y\|$.

(iii) $Y$ is invariant under left and right translations, i.e. $L_{x} Y \subseteq Y$ and $R_{x} Y \subseteq Y$ for all $x \in \mathcal{G}$. If we set $u(x)=\left\|L_{x} \mid Y\right\|$ and $v(x)=$ $\Delta\left(x^{-1}\right)\left\|R_{x^{-1}} \mid Y\right\|$, the operator norms of translations on $Y$, then we require that

$$
L_{u}^{1} * Y \subseteq Y \text { and } Y * L_{v}^{1} \subseteq Y .
$$

We only work with pairs $(Y, w)$, where the weight function $w$ on $\mathcal{G}$ satisfies

$$
\begin{aligned}
& w(x) \geq C \max \left\{u(x), u\left(x^{-1}\right), v(x), \Delta\left(x^{-1}\right) v\left(x^{-1}\right)\right\}, \\
& w(x)=w\left(x^{-1}\right) \Delta\left(x^{-1}\right)
\end{aligned}
$$

for some constant $C>0$. In particular, $w(x) \geq 1,\left\|f\left|L_{w}^{1}\|=\| f^{\vee}\right| L_{w}^{1}\right\|$ and $Y * L_{w}^{1} \subset Y$.

We emphasize that the assumptions in coorbit theory concern mostly the weight $w$ associated to $Y$; the main results hold simultaneously for the entire class of function spaces $Y$ with the same weight $w$, and not just for a single $Y$.

The Lebesgue spaces $L^{p}(\mathcal{G}), 1 \leq p \leq \infty$, and the mixed-norm spaces $L^{p, q}(\mathcal{G})$ provide some natural examples of solid Banach spaces on $\mathcal{G}$. If $w$ is some positive measurable weight function on $\mathcal{G}$, then we define $L_{w}^{p}$ to be the set of all measurable function $F$ such that $F w \in L^{p}$ with $\left\|F \mid L_{w}^{p}\right\|:=$ 
$\left\|F w \mid L^{p}\right\|$. A continuous weight $w$ is called submultiplicative if $w(x y) \leq$ $w(x) w(y)$ for all $x, y \in \mathcal{G}$. A weight function $m$ is called $w$-moderate if $m(x y z) \leq C w(x) m(y) w(z), x, y, z \in \mathcal{G}$. It follows that $L_{m}^{p}$ is invariant under left and right translations if and only if $m$ is $w$-moderate.

As a next ingredient, we need certain discrete sets in $\mathcal{G}$. Let $X=\left(x_{i}\right)_{i \in I}$ be a discrete set of points in $\mathcal{G}$, and $U$ a relatively compact neighborhood of $e$ in $\mathcal{G}$. Then $X$ is called:

(a) $U$-dense if $\mathcal{G}=\bigcup_{i \in I} x_{i} U$;

(b) relatively separated if for all compact sets $K \subset \mathcal{G}$ there exists a constant $C_{K}$ such that $\sup _{j \in I} \#\left\{i \in I: x_{i} K \cap x_{j} K \neq \emptyset\right\} \leq C_{K}$ :

(c) well-spread if it is both relatively separated and $U$-dense for some $U$.

Definition 2.1. Given a well-spread family $X=\left(x_{i}\right)_{i \in I}$, and a relatively compact neighborhood $U$ of $e \in \mathcal{G}$, we define the sequence space $Y_{d}$ associated to a solid Banach function space $Y$ to be

$$
Y_{d}:=Y_{d}(X):=Y_{d}(X, U):=\left\{\left(c_{i}\right)_{i \in I}: \sum_{i \in I} c_{i} \chi_{x_{i} U} \in Y\right\},
$$

endowed with the norm $\left\|\left(c_{i}\right)_{i \in I}\left|Y_{d}\|:=\| \sum_{i \in I} c_{i} \chi_{x_{i} U}\right| Y\right\|$.

For instance, if $Y=L_{w}^{p}(\mathcal{G})$, then $Y_{d}=L_{w}^{p}(\mathcal{G})_{d}=\ell_{\widetilde{w}}^{p}$, where $\widetilde{w}$ is determined by $\widetilde{w}_{i}=w\left(x_{i}\right)$.

If $L_{0}^{\infty}(\mathcal{G})$ is dense in $Y$, then the finite sequences are dense in $Y_{d}[6$, Lemma 3.5(a)].

2.3. Wiener amalgam spaces. Let $U$ be some relatively compact neighborhood of $e \in \mathcal{G}$. We define the local maximum function of $F$ by

$$
F_{\sharp}(x):=\sup _{y \in x U}|F(y)|, \quad x \in \mathcal{G},
$$

whenever $F$ is locally bounded, in symbols $F \in L_{\text {loc }}^{\infty}$. Given a Banach space $Y$ of functions on $\mathcal{G}$ satisfying 2.2(i)-(iii), the Wiener amalgam space $W\left(L^{\infty}, Y\right)$ is defined by

$$
W\left(L^{\infty}, Y\right):=\left\{F \in L_{\mathrm{loc}}^{\infty}: F_{\sharp} \in Y\right\}
$$

and equipped with the norm

$$
\left\|F\left|W\left(L^{\infty}, Y\right)\|:=\| F_{\sharp}\right| Y\right\| .
$$

Similarly, the right local maximum function is $F_{\sharp}^{R}(x)=\sup _{y \in U^{-1} x^{-1}}|F(y)|$ and the right Wiener amalgam space $W^{R}\left(L^{\infty}, Y\right)$ is defined by the norm $\left\|F\left|W^{R}\left(L^{\infty}, Y\right)\|:=\| F_{\sharp}^{R}\right| Y\right\|$. By $W^{R}(C, Y)$ we denote the closed subspace of $W^{R}\left(L^{\infty}, Y\right)$ consisting of continuous functions. In several arguments we need the following convolution relation from [6, Proposition 5.2]. 
Proposition 2.2. If $\left(c_{i}\right)_{i \in I} \in Y_{d}$ and $H \in W^{R}\left(L^{\infty}, L_{w}^{1}\right)$, then the sum $\sum_{i \in I} c_{i} L_{x_{i}} H$ is in $Y$ and

$$
\left\|\sum_{i \in I} c_{i} L_{x_{i}} H\left|Y\|\leq C\|\left(c_{i}\right)_{i \in I}\right| Y_{d}\right\|\left\|H \mid W^{R}\left(L^{\infty}, L_{w}^{1}\right)\right\| .
$$

The sum $\sum_{i \in I} c_{i} L_{x_{i}} H$ converges unconditionally in $Y$ if $L_{0}^{\infty}$ is dense in $Y$, and otherwise converges $w^{*}$ in the $\sigma\left(Y, L_{w}^{1}\right)$-topology.

2.4. Coorbit spaces. Let $\pi$ be an irreducible unitary representation of $\mathcal{G}$ on a Hilbert space $\mathcal{H}$. For a fixed $g \in \mathcal{H}$, the abstract wavelet transform is defined as

$$
V_{g} f(x):=\langle f, \pi(x) g\rangle, \quad f \in \mathcal{H}, x \in \mathcal{G} .
$$

The representation $\pi$ is called square-integrable if there is a non-zero vector $g \in \mathcal{H}$, called an admissible vector, such that $V_{g} g \in L^{2}(\mathcal{G})$. The main ingredient in coorbit space theory is a reproducing formula of the form

$$
V_{g} f=V_{g} f * V_{g} g \quad \text { for all } f \in \mathcal{H},
$$

where $*$ denotes the convolution on $\mathcal{G}$. Reproducing formulae are known to hold for many types of representations. In particular, (6) holds for every square-integrable, irreducible representation $\pi$ of $\mathcal{G}$ [21] and also for many reducible square-integrable representations (see $[12,19])$. In order to introduce the coorbit spaces we first need to extend the definition of the abstract wavelet transform to a suitable space of distributions. We define the class of analyzing vectors,

$$
\mathbb{A}_{w}:=\left\{g \in \mathcal{H}: V_{g} g \in L_{w}^{1}\right\} .
$$

Let us assume that $\mathbb{A}_{w}$ is non-trivial, i.e., $\pi$ is integrable; then $\pi$ is also square-integrable. For a fixed $g \in \mathbb{A}_{w} \backslash\{0\}$ we define

$$
\mathcal{H}_{w}^{1}:=\left\{f \in \mathcal{H}: V_{g} f \in L_{w}^{1}\right\}
$$

endowed with the norm $\left\|f\left|\mathcal{H}_{w}^{1}\|:=\| V_{g} f\right| L_{w}^{1}\right\|$. Further, we denote by $\left(\mathcal{H}_{w}^{1}\right)^{\urcorner}$the anti-dual, i.e., the space of all bounded conjugate-linear functionals on $\mathcal{H}_{w}^{1}$. An equivalent norm on $\left(\mathcal{H}_{w}^{1}\right)^{\urcorner}$is given by $\left\|V_{g} f \mid L_{1 / w}^{\infty}\right\|$. Since the inner product on $\mathcal{H} \times \mathcal{H}$ extends to a sesquilinear form on $\left.\left(\mathcal{H}_{w}^{1}\right)\right\urcorner \times \mathcal{H}_{w}^{1}$, the extended representation coefficients

$$
\left.V_{g} f(x)=\langle f, \pi(x) g\rangle, \quad f \in\left(\mathcal{H}_{w}^{1}\right)\right\urcorner, g \in \mathbb{A}_{w},
$$

are well-defined. We are now in a position to define coorbit spaces.

Definition 2.3. Let $Y$ be a solid Banach space of functions on $\mathcal{G}$ with canonical weight $w$. Then for $g \in \mathbb{A}_{w}, g \neq 0$, the coorbit space is defined by

$$
\text { Co } Y:=\left\{f \in\left(\mathcal{H}_{w}^{1}\right)^{\urcorner}: V_{g} f \in Y\right\}
$$

with the norm $\left\|f\left|\operatorname{Co} Y\|:=\| V_{g} f\right| Y\right\|$. 
REMARK 2.4. $\mathcal{H}_{w}^{1},\left(\mathcal{H}_{w}^{1}\right)^{\urcorner}$, and Co $Y$ are $\pi$-invariant Banach spaces. If $\pi$ is irreducible, then their definitions do not depend on the choice of the analyzing vector $g$ in the sense that different windows provide equivalent norms [6, Thm. 4.2].

2.5. Atomic decomposition and Banach frames. Next we describe atomic decompositions and Banach frames in coorbit spaces as outlined in $[6,7,15]$. The treatment of coherent frames for $\operatorname{Co} Y$ requires a further restriction of the set of analyzing vectors. The set of "better vectors" is given by

$$
\mathbb{B}_{w}:=\left\{g \in \mathcal{H}: V_{g} g \in W^{R}\left(L^{\infty}, L_{w}^{1}\right)\right\} .
$$

If $\mathbb{A}_{w} \neq \emptyset$, then also $\mathbb{B}_{w} \neq \emptyset$.

Below we summarize the results about the existence of atomic decompositions and frames from [15, Theorem U].

THEOREM 2.5. Let $Y$ satisfy 2.2(i)-(iii) with canonical weight $w$ given by (1) and assume that $g \in \mathbb{B}_{w}, g \neq 0$. Then there exists a neighborhood $U$ of e such that for any $U$-dense and relatively separated family $X=\left(x_{i}\right)_{i \in I}$ in $\mathcal{G}$ the set $\left\{\pi\left(x_{i}\right) g\right\}_{i \in I}$ provides an atomic decomposition and a Banach frame for Co $Y$.

(A) (Atomic decomposition) Every $f \in \operatorname{Co} Y$ has an expansion

$$
f=\sum_{i \in I} c_{i}(f) \pi\left(x_{i}\right) g
$$

where the sequence of coefficients $\left(c_{i}(f)\right)_{i \in I}$ depends linearly on $f$ and satisfies

$$
\left\|\left(c_{i}(f)\right)_{i \in I}\left|Y_{d}\|\leq C\| f\right| \operatorname{Co} Y\right\|
$$

with a constant $C$ depending only on $g$. Conversely, if $\left(c_{i}\right)_{i \in I} \in Y_{d}$, then $f=\sum_{i \in I} c_{i} \pi\left(x_{i}\right) g$ is in Co $Y$ and

$$
\left\|f\left|\operatorname{Co} Y\left\|\leq C^{\prime}\right\|\left(c_{i}\right)_{i \in I}\right| Y_{d}\right\| \text {. }
$$

The series defining $f$ converges unconditionally in the norm of $\mathrm{Co} Y$ if $L_{0}^{\infty}(\mathcal{G})$ is dense in $Y$, otherwise it converges unconditionally in the weak $k^{*}$ topology of $\left.\left(\mathcal{H}_{w}^{1}\right)\right\urcorner$.

(B) (Banach frames) $\left\{\pi\left(x_{i}\right) g\right\}_{i \in I}$ is a Banach frame for Co $Y$. This means that

(i) There are two constants $C_{1}, C_{2}>0$ depending only on $g$ such that

$$
C_{1}\left\|f\left|\operatorname{Co} Y\|\leq\|\left(\left\langle f, \pi\left(x_{i}\right) g\right\rangle\right)_{i \in I}\right| Y_{d}\right\| \leq C_{2}\|f \mid \operatorname{Co} Y\| .
$$

(ii) (Reconstruction operator) There exists a bounded mapping $R$ from $Y_{d}(X)$ onto $\operatorname{Co} Y$ such that $f=R\left(\left\langle f, \pi\left(x_{i}\right) g\right\rangle_{i \in I}\right)$. 
(C) (Dual frames) There exists a "dual frame" $\left\{e_{i}\right\}_{i \in I}$ in $\mathcal{H}_{w}^{1}$ such that, for every $f \in \operatorname{Co} Y$,

$$
f=\sum_{i \in I}\left\langle f, e_{i}\right\rangle \pi\left(x_{i}\right) g
$$

and $\left\|\left(\left\langle f, e_{i}\right\rangle\right)_{i \in I} \mid Y_{d}\right\|$ is an equivalent norm on $\operatorname{Co} Y$.

3. Molecules in coorbit space theory. In this section we introduce the notion of molecules in coorbit spaces and state and prove our main result.

Definition 3.1. Assume that $g \in \mathbb{B}_{w}, g \neq 0$, and let $X=\left(x_{i}\right)_{i \in I}$ be a well-spread family in $\mathcal{G}$. A collection of functions $\left\{m_{i}\right\}_{i \in I} \subset \mathcal{H}$ is called a set of molecules if there exists an envelope function $H \in W^{R}\left(L^{\infty}, L_{w}^{1}\right)$ such that

$$
\left|V_{g} m_{i}(z)\right| \leq L_{x_{i}} H(z), \quad i \in I .
$$

REMARK 3.2. We may think of $\mathcal{G}$ as a kind of phase space and the function $V_{g} f$ (for fixed $g \neq 0$ ) as a phase-space representation of $f$. The molecule $m_{i}$ is then localized at $x_{i} \in \mathcal{G}$ and a set of molecules has a uniform envelope in phase space. In other words, each molecule has the same phasespace concentration.

EXAmple 3.3. 1. Every set of atoms $\left\{\pi\left(x_{i}\right) g\right\}_{i \in I}$ for $g \in \mathbb{B}_{w}$ is a set of molecules in the sense of Definition 3.1, because $\left|\left\langle\pi\left(x_{i}\right) g, \pi(z) g\right\rangle\right|=$ $\left|\left\langle g, \pi\left(x_{i}^{-1} z\right) g\right\rangle\right|=L_{x_{i}}\left|V_{g} g(z)\right|$ and $V_{g} g \in W^{R}\left(L^{\infty}, L_{w}^{1}\right)$.

2. Fix $g_{0} \in \mathbb{B}_{w}$ and a positive function $H \in W^{R}\left(L^{\infty}, L_{w}^{1}\right)$, and set

$$
C_{H}:=\left\{g \in \mathcal{H}:\left|V_{g_{0}} g(x)\right| \leq H(x)\right\} .
$$

If $X=\left(x_{i}\right)_{i \in I}$ is well-spread and $g_{i} \in C_{\mathcal{H}}$, then

$$
\left|V_{g_{0}}\left(\pi\left(x_{i}\right) g_{i}\right)(z)\right|=\left|\left\langle\pi\left(x_{i}\right) g_{i}, \pi(z) g_{0}\right\rangle\right|=\left|L_{x_{i}} V_{g_{0}} g(z)\right| \leq L_{x_{i}} H(z),
$$

and so the set $\left\{m_{i}=\pi\left(x_{i}\right) g_{i}\right\}_{i \in I}$ forms a family of $H$-molecules.

In preparation for the main result, we verify the following basic properties of molecules.

LEMMA 3.4.

(i) The definition of molecules does not depend on the particular choice of the window $g \in \mathbb{B}_{w}$.

(ii) (Synthesis) Let $\left\{m_{i}\right\}_{i \in I}$ be a set of molecules subordinated to $H \in$ $W^{R}\left(L^{\infty}, L_{w}^{1}\right)$. The synthesis operator $\left(c_{i}\right)_{i \in I} \mapsto \sum_{i \in I} c_{i} m_{i}$ is bounded from $Y_{d}$ to $\operatorname{Co} Y$. If $\left(c_{i}\right)_{i \in I} \in Y_{d}$, then $f=\sum_{i \in I} c_{i} m_{i} \in \operatorname{Co} Y$ and

$$
\left\|\sum_{i \in I} c_{i} m_{i}\left|\operatorname{Co} Y\|\leq C\|\left(c_{i}\right)_{i \in I}\right| Y_{d}\right\|\left\|H \mid W^{R}\left(L^{\infty}, L_{w}^{1}\right)\right\|
$$


for some constant $C$. The sum defining $f$ converges unconditionally whenever $L_{0}^{\infty}(\mathcal{G})$ is dense in $Y$, and in the $w^{*}$-sense on $\left(\mathcal{H}_{w}^{1}\right)^{\urcorner}$ otherwise.

(iii) (Analysis) If, in addition, $H \in W\left(L^{\infty}, L_{w}^{1}\right)$, then the coefficient operator $C f:=\left(\left\langle f, m_{i}\right\rangle\right)_{i \in I}$ is bounded from $\operatorname{Co} Y$ to $Y_{d}$ with

$$
\left\|\left(\left\langle f, m_{i}\right\rangle\right)_{i \in I}\left|Y_{d}\|\leq C\| f\right| \operatorname{Co} Y\right\| .
$$

Proof. To prove (i) we assume that $g, h \in \mathbb{B}_{w}$ and that $\left\{\pi\left(z_{j}\right) g: j \in J\right\}$ is a (Banach) frame for $\mathcal{H}_{w}^{1}$. After substituting the frame expansion of $h=$ $\sum_{j \in J}\left\langle h, e_{j}\right\rangle \pi\left(z_{j}\right) g$, where the sequence $\left(c_{j}\right)_{j \in I}$ with $c_{j}:=\left|\left\langle h, e_{j}\right\rangle\right|$ is in $\ell_{\widetilde{w}}^{1}$, into (8) we obtain

$$
\begin{aligned}
\left|V_{h} m_{i}(z)\right| & \left.=\mid\left\langle m_{i}, \pi(z) h\right\rangle\right)\left|\leq \sum_{j \in I}\right|\left\langle h, e_{j}\right\rangle||\left\langle m_{i}, \pi(z) \pi\left(z_{j}\right) g\right\rangle \mid \\
& \leq \sum_{j \in I} c_{j} L_{x_{i}} H\left(z z_{j}\right)=L_{x_{i}}\left(\sum_{j \in I} c_{j} R_{z_{j}} H(z)\right) .
\end{aligned}
$$

Since $W^{R}\left(L^{\infty}, L_{w}^{1}\right)$ is invariant under right translations, we find that $\widetilde{H}=$ $\sum_{j \in I} c_{j} R_{z_{j}} H \in W^{R}\left(L^{\infty}, L_{w}^{1}\right)$, and (8) is satisfied for $h$ in place of $g$ with the envelope function $\widetilde{H}$.

For the proof of (ii) we assume first that $L_{0}^{\infty}(\mathcal{G})$ is dense in $Y$. In this case, the finite sequences are dense in $Y_{d}$ by [6, Lemma 3.5] and thus it suffices to prove (3.4) for finite sequences. If $\operatorname{supp}(c)$ is finite, then by the solidity of $Y$ and the property of molecules we obtain

$$
\begin{aligned}
\left\|\sum_{i \in I} c_{i} m_{i} \mid \operatorname{Co} Y\right\| & =\left\|V_{g}\left(\sum_{i \in I} c_{i} m_{i}\right)\left|Y\|\leq\| \sum_{i \in I}\right| c_{i}|| V_{g} m_{i}|| Y\right\| \\
& \leq\left\|\sum_{i \in I}\left|c_{i}\right| L_{x_{i}} H\left|Y\|\leq C\|\left(c_{i}\right)_{i \in I}\right| Y_{d}\right\|\left\|H \mid W^{R}\left(L^{\infty}, L_{w}^{1}\right)\right\| .
\end{aligned}
$$

The last inequality above follows immediately from Proposition 2.2. This norm estimate also implies the unconditional convergence in Co $Y$.

If $L_{0}^{\infty}(\mathcal{G})$ is not dense in $Y$, then still $\sum_{i \in I}\left|c_{i}\right| L_{x_{i}} H \in Y$, but the sum converges only in the weak* sense. Thus the above estimate still holds, and $\sum_{i \in I} c_{i} m_{i} \in \operatorname{Co} Y$ is $w^{*}$-convergent.

Finally, we show (iii). By virtue of Theorem 2.5, every $f \in \operatorname{Co} Y$ has an expansion

$$
f=\sum_{j \in J} c_{j} \pi\left(z_{j}\right) g
$$

with $\left(c_{j}\right)_{j \in J} \in Y_{d}$ and $\left\|\left(c_{j}\right)_{j \in J}\left|Y_{d}\|\leq C\| f\right| \operatorname{Co} Y\right\|$. Plugging in again the above expansion yields

$$
\left|\left\langle f, m_{i}\right\rangle\right| \leq \sum_{j \in J}\left|c_{j}\right|\left|\left\langle\pi\left(z_{j}\right) g, m_{i}\right\rangle\right| \leq \sum_{j \in J} c_{j} H\left(x_{i}^{-1} z_{j}\right)=\sum_{j \in J} c_{j} L_{z_{j}} H^{\vee}\left(x_{i}\right) .
$$


Consequently, we get

$$
\begin{aligned}
\left\|\left(\left\langle f, m_{i}\right\rangle\right)_{i \in I} \mid Y_{d}\right\| & \leq\left\|\sum_{j \in J} c_{j} L_{z_{j}} H^{\vee} \mid W\left(L^{\infty}, Y\right)\right\| \\
& \leq\left\|\left(c_{i}\right)_{i \in I}\left|Y_{d}\|\| H^{\vee}\right| W^{R}\left(L^{\infty}, L_{w}^{1}\right)\right\| \leq C\|f \mid \operatorname{Co} Y\| .
\end{aligned}
$$

Now we formulate our main result on the boundedness of operators on coorbit spaces.

Theorem 3.5. Suppose that $g \in \mathbb{B}_{w}$ and that $\left\{\pi\left(x_{i}\right) g\right\}_{i \in I}$ forms a Banach frame for Co $Y$ with canonical dual frame $\left\{e_{i}\right\}_{i \in I}$ (as guaranteed by Theorem 2.5). Assume that the operator $T$ is bounded from $\mathcal{H}_{w}^{1}$ to $\left.\left(\mathcal{H}_{w}^{1}\right)\right\urcorner$ and that $T$ maps the atoms $\pi\left(x_{i}\right) g, i \in I$, to the molecules $m_{i}=T\left(\pi\left(x_{i}\right) g\right)$ with envelope $H \in W^{R}\left(L^{\infty}, L_{w}^{1}\right)$. Then $T$ extends to a bounded operator on Co $Y$. Furthermore, the operator norm of $T$ is bounded by $\left\|H \mid W^{R}\left(L^{\infty}, L_{w}^{1}\right)\right\|$.

Proof. We would like to define $T f=\sum_{i \in I} c_{i} T\left(\pi\left(x_{i}\right) g\right)=\sum_{i \in I} c_{i} m_{i}$ for $f=\sum_{i \in I} c_{i} \pi\left(x_{i}\right) g$. Lemma 3.4 then yields the correct norm estimates. However, in general, the representation of $f$ with respect to $\left\{\pi\left(x_{i}\right) g\right\}$ is not unique, therefore we have to show that the natural extension procedure is unique.

Step 1. First we define a canonical extension $\widetilde{T}$ of $T$ to Co $Y$ via the frame expansion of $f$. Let $e_{i} \in \mathcal{H}_{w}^{1}$ be the dual frame of $\pi\left(x_{i}\right) g, i \in I$, the existence of which is asserted in Theorem 2.5(C). Then $f \in$ Co $Y$ has the expansion $f=\sum_{i \in I}\left\langle f, e_{i}\right\rangle \pi\left(x_{i}\right) g$ with coefficient sequence $\left(\left\langle f, e_{i}\right\rangle\right)_{i \in I} \in Y_{d}$ and

$$
\left\|\left(\left\langle f, e_{i}\right\rangle\right)_{i \in I}\left|Y_{d}\|\leq C\| f\right| \operatorname{Co} Y\right\|,
$$

where the constant $C>0$ is independent of $f$. We define $\widetilde{T} f$ by

$$
\widetilde{T} f=\sum_{i \in I}\left\langle f, e_{i}\right\rangle T\left(\pi\left(x_{i}\right) g\right)=\sum_{i \in I}\left\langle f, e_{i}\right\rangle m_{i} .
$$

By Lemma 3.4(ii) we find that $\widetilde{T} f$ is in Co $Y$ and that

$$
\begin{aligned}
\|\widetilde{T} f \mid \operatorname{Co} Y\| & \leq C\left\|\left(\left\langle f, e_{i}\right\rangle\right)_{i \in I}\left|Y_{d}\|\| H\right| W^{R}\left(L^{\infty}, L_{w}^{1}\right)\right\| \\
& \leq C^{\prime}\left\|H\left|W^{R}\left(L^{\infty}, L_{w}^{1}\right)\|\| f\right| \operatorname{Co} Y\right\| .
\end{aligned}
$$

Furthermore, the series defining $\widetilde{T} f$ converges unconditionally in Co $Y$ if $L_{0}^{\infty}$ is dense in $Y$, and $w^{*}$ in $\left(\mathcal{H}_{w}^{1}\right)^{\urcorner}$otherwise.

STEP 2. It remains to show that $\widetilde{T}$ coincides with $T$ on $\mathcal{H}_{w}^{1}$. Here we exploit the assumed continuity of $T$ from $\mathcal{H}_{w}^{1}$ to $\left.\left(\mathcal{H}_{w}^{1}\right)\right\urcorner$. This means that the convergence $f_{n} \rightarrow f$ in $\mathcal{H}_{w}^{1}$ implies the $w^{*}$-convergence $T f_{n} \rightarrow T f$. In 
particular, the net of partial sums

$$
f_{F}=\sum_{k \in F}\left\langle\pi\left(x_{i}\right) g, e_{k}\right\rangle \pi\left(x_{k}\right) g
$$

converges to $\pi\left(x_{i}\right) g$ as $F \rightarrow I$, where $(F)$ is the net of finite subsets of $I$ ordered by inclusion. Consequently,

$$
\begin{aligned}
m_{i} & =T\left(\pi\left(x_{i}\right) g\right)=w^{*}-\lim _{F \rightarrow I} T f_{F} \\
& =w^{*}-\lim _{F \rightarrow I} \sum_{k \in F}\left\langle\pi\left(x_{i}\right) g, e_{k}\right\rangle T\left(\pi\left(x_{k}\right) g\right) \\
& =w^{*}-\lim _{F \rightarrow I} \sum_{k \in F}\left\langle\pi\left(x_{i}\right) g, e_{k}\right\rangle m_{k}=\widetilde{T}\left(\pi\left(x_{i}\right) g\right) .
\end{aligned}
$$

Since the $m_{k}$ 's are molecules, the series in (13) converges also in $\mathcal{H}_{w}^{1}$ by Lemma 3.4. The identity $T\left(\pi\left(x_{i}\right) g\right)=\widetilde{T}\left(\pi\left(x_{i}\right) g\right)$ implies that $T f=\widetilde{T} f$ whenever $f=\sum_{i \in I} c_{i} \pi\left(x_{i}\right) g$ and $\left(c_{i}\right)_{i \in I} \in \ell_{w}^{1}$.

We now take $\widetilde{T}$ as the desired extension of $T$ from $\mathcal{H}_{w}^{1}$ to Co $Y$. By Step 1 this extension is bounded on Co $Y$. This completes the proof.

REMARK 3.6. We observe that Theorem 3.5 asserts the simultaneous boundedness of $T$ on all coorbit spaces Co $Y$ with the same associated weight $w$ given in (1).

REMARK 3.7. Theorem 3.5 can probably be formulated for quasi-Banach spaces as well by using Rauhut's extension of coorbit space theory [24].

\section{Examples and applications}

4.1. The Heisenberg group and time-frequency molecules. We now describe the consequences of Theorem 3.5 in the context of time-frequency molecules. Time-frequency molecules were introduced in [17, Section 5.3] and independently in [1] and were studied in detail in [20, Section 7].

We first discuss how the modulation spaces fit into coorbit space setting. We consider the $d$-dimensional reduced Heisenberg group $\mathcal{G}_{\mathrm{H}}=\mathbb{R}^{d} \times \mathbb{R}^{d} \times \mathbb{T}$ with multiplication

$$
(x, \omega, \tau)\left(x^{\prime}, \omega^{\prime}, \tau^{\prime}\right)=\left(x+x^{\prime}, \omega+\omega^{\prime}, \tau \tau^{\prime} e^{\pi i\left(x^{\prime} \cdot \omega-x \cdot \omega^{\prime}\right)}\right) .
$$

Let $T_{x} f(t)=f(t-x)$ and $M_{\omega} f(t)=e^{2 \pi i t \cdot \omega} f(t)$ be the operators of translation and modulation, respectively, and $\pi$ be the Schrödinger representation of $\mathcal{G}_{\mathrm{H}}$ acting on $L^{2}\left(\mathbb{R}^{d}\right)$ by time-frequency shifts

$$
\pi(x, \omega, \tau):=\tau e^{\pi i x \cdot \omega} T_{x} M_{\omega}=\tau e^{-\pi i x \cdot \omega} M_{\omega} T_{x} .
$$

This is an irreducible, unitary and square-integrable representation of $\mathcal{G}_{\mathrm{H}}$. Except for a trivial phase factor the representation coefficient $V_{g} f(x, \omega, \tau)=$ 
$\langle f, \pi(x, \omega, \tau) g\rangle_{L^{2}\left(\mathbb{R}^{d}\right)}$ coincides with the Short-Time Fourier Transform (STFT) given by

$$
\operatorname{STFT}_{g} f(x, \omega)=\left\langle f, M_{\omega} T_{x} g\right\rangle_{L^{2}\left(\mathbb{R}^{d}\right)}=\int_{\mathbb{R}^{d}} f(t) \overline{g(t-x)} e^{-2 \pi i \omega \cdot t} d t,
$$

whenever the integral makes sense. Otherwise, we fix $g \in \mathcal{S}\left(\mathbb{R}^{d}\right)$ and extend the STFT to tempered distributions $\mathcal{S}^{\prime}\left(\mathbb{R}^{d}\right)$ by interpreting the bracket $\langle f, g\rangle$ as a dual pairing between an element $f \in \mathcal{S}^{\prime}\left(\mathbb{R}^{d}\right)$ and $g \in \mathcal{S}\left(\mathbb{R}^{d}\right)$. For more information on the STFT the reader is referred to [16].

We take the liberty to drop the center $\{0\} \times\{0\} \times \mathbb{T}$ of $\mathcal{G}_{\mathrm{H}}$ and consider function spaces on $\mathbb{R}^{2 d}$ instead of $\mathcal{G}_{\mathrm{H}}$. As a standard example we take the mixed-norm spaces $L_{m}^{p, q}\left(\mathbb{R}^{2 d}\right)$ for $1 \leq p, q \leq \infty$ and some $w$-moderate weight function $m$ on $\mathbb{R}^{2 d}$ with the norm

$$
\left\|F \mid L_{m}^{p, q}\left(\mathbb{R}^{2 d}\right)\right\|:=\left(\int_{\mathbb{R}^{d}}\left(\int_{\mathbb{R}^{d}}|F(x, \omega)|^{p} m(x, \omega)^{p} d x\right)^{q / p} d \omega\right)^{1 / q} .
$$

The modulation spaces are obtained as the coorbits of $L_{m}^{p, q}\left(\mathbb{R}^{2 d}\right)$ with respect to the Schrödinger representation $\pi$,

$$
M_{m}^{p, q}\left(\mathbb{R}^{d}\right)=\operatorname{Co} L_{m}^{p, q}\left(\mathbb{R}^{2 d}\right)=\left\{f \in \mathcal{S}^{\prime}\left(\mathbb{R}^{d}\right): \operatorname{STFT}_{g} f \in L_{m}^{p, q}\left(\mathbb{R}^{2 d}\right)\right\}
$$

for fixed non-zero $g \in \mathcal{S}\left(\mathbb{R}^{d}\right)$. For the Heisenberg group many technical subtleties of the general set-up of coorbit space theory disappear. For instance $W\left(L^{\infty}, L_{w}^{1}\right)\left(\mathcal{G}_{\mathrm{H}}\right)=W^{R}\left(L^{\infty}, L_{w}^{1}\right)\left(\mathcal{G}_{\mathrm{H}}\right)$ and

$$
\mathbb{B}_{w}=\mathbb{A}_{w}=M_{w}^{1,1}\left(\mathbb{R}^{2 d}\right)
$$

(cf. [7, Lemma 7.2]). As long as $w$ and $m$ have polynomial growth, one may use the Schwartz class $\mathcal{S}\left(\mathbb{R}^{d}\right) \subset \mathbb{A}_{w}$ as a convenient space of test functions.

In the context of modulation spaces and the Heisenberg group, the natural discrete sets are lattices, i.e., discrete co-compact subgroups of the form $\Lambda=A \mathbb{Z}^{2 d}$ for some invertible $2 d \times 2 d$-matrix $A$. Let $G(g, \Lambda):=\{\pi(\lambda) g$ : $\lambda \in \Lambda\}$ be the orbit of $g$ under $\Lambda$ (a so-called Gabor system).

Given a symbol $\sigma \in \mathcal{S}^{\prime}\left(\mathbb{R}^{2 d}\right)$, the pseudodifferential operator $\sigma^{w}$ is informally given by

$$
\sigma^{w} f=\iint_{\mathbb{R}^{d}} \widehat{\sigma}(\xi, u) e^{-\pi i \xi u} T_{-u} M_{\xi} f d u d \xi,
$$

whenever the integral makes sense, otherwise it is interpreted in the weak sense. The mapping $\sigma \mapsto \sigma^{w}$ is called the Weyl transform.

The abstract Definition 3.1 can be rephrased as follows (cf. [17] and [20, Definition 7.1]).

Definition 4.1. Fix a non-zero $g \in M_{w}^{1,1}\left(\mathbb{R}^{d}\right)$. A collection $\left\{m_{\lambda}\right\}_{\lambda \in \Lambda}$ of functions forms a set of time-frequency molecules if there exists a function 
$H \in W\left(L^{\infty}, L_{w}^{1}\right)\left(\mathbb{R}^{2 d}\right)$ such that

$$
\left|\left\langle m_{\lambda}, \pi(z) g\right\rangle\right| \leq H(z-\lambda), \quad \lambda \in \Lambda .
$$

In our language, the main theorem of [18] (cf. also [20, Proposition 7.1]) can be formulated as follows. [We write $j$ for the rotation mapping $j\left(z_{1}, z_{2}\right)=$ $\left(z_{2},-z_{1}\right)$ with $\left(z_{1}, z_{2}\right) \in \mathbb{R}^{2 d}$.]

Proposition 4.2. Fix a non-zero $g \in M_{w}^{1,1}$ and suppose that $\mathcal{G}(g, \Lambda)$ is a Gabor frame for $L^{2}\left(\mathbb{R}^{d}\right)$. Then the following are equivalent.

(i) $\sigma \in M_{w \circ j^{-1}}^{\infty, 1}\left(\mathbb{R}^{2 d}\right)$.

(ii) There exists a function $H \in L_{w}^{1}\left(\mathbb{R}^{2 d}\right)$ such that

$$
\left|\left\langle\sigma^{w} \pi(w) g, \pi(z) g\right\rangle\right| \leq H(z-w), \quad w, z \in \mathbb{R}^{2 d} .
$$

(iii) There is a function $H \in W\left(L^{\infty}, L_{w}^{1}\right)\left(\mathbb{R}^{2 d}\right)$ such that the corresponding pseudodifferential operator $\sigma^{w}$ maps the time-frequency shifts $\{\pi(\lambda) g\}$ to time-frequency molecules $\left\{m_{\lambda}\right\}_{\lambda \in \Lambda}$ in the sense of Definition 4.1 with envelope function $H$.

Proof. The equivalence (i) $\Leftrightarrow$ (ii) was proved in [17, Thm. 3.2].

(ii) $\Leftrightarrow$ (iii). Let $m_{\lambda}=\sigma^{w}(\pi(\lambda) g)$. Then by (ii) we have $\left|\left\langle m_{\lambda}, \pi(z) g\right\rangle\right| \leq$ $H(z-\lambda)$. At first $H$ is only in $L_{w}^{1}\left(\mathbb{R}^{2 d}\right)$, but it was shown in [17, Thm. 3.2] that the envelope function $H$ can be chosen in $W\left(L^{\infty}, L_{w}^{1}\right)$. Thus the set of $m_{\lambda}, \lambda \in \Lambda$, is a set of time-frequency molecules in the sense of Definition 4.1.

Conversely, if $m_{\lambda}=\sigma^{w}(\pi(\lambda) g)$ is a set of molecules, then we have $\left|\left\langle\sigma^{w}(\pi(\lambda) g), \pi(\mu) g\right\rangle\right| \leq H(\mu-\lambda)$. Again, by [18] this property implies that $\sigma \in M_{w \circ j^{-1}}^{\infty, 1}\left(\mathbb{R}^{2 d}\right)$.

Since the modulation spaces are the coorbit spaces for the Schrödinger representation, Theorem 3.5 now implies the boundedness of pseudodifferential operators with symbol in $M_{w o j^{-1}}^{\infty}\left(\mathbb{R}^{2 d}\right)$ on a large class of modulation spaces. See [16, Thm. 14.5.6] and [26] for different proofs.

COROLlary 4.3. If $\sigma \in M_{w \circ j^{-1}}^{\infty, 1}\left(\mathbb{R}^{2 d}\right)$, then $\sigma^{w}$ is bounded simultaneously on all modulation spaces $M_{m}^{p, q}\left(\mathbb{R}^{d}\right)$ for $1 \leq p, q \leq \infty$ and every $w$ moderate weight function $m$.

4.2. The affine group and time-scale molecules. We next consider the affine group $\mathcal{G}_{\mathrm{A}}=\mathbb{R}^{d} \times \mathbb{R}_{+}$with multiplication $(x, s) \cdot\left(x^{\prime}, s^{\prime}\right)=\left(x+s x^{\prime}, s s^{\prime}\right)$ for $x, x^{\prime} \in \mathbb{R}^{d}$ and $s, s^{\prime}>0$. Let the dilation operator be given by $D_{s} f(x)=$ $s^{-d / 2} f\left(s^{-1} x\right)$ with $s>0$. A unitary representation of $\mathcal{G}_{\mathrm{A}}$ acts on $L^{2}\left(\mathbb{R}^{d}\right)$ by translations and dilations:

$$
\pi(x, s) g(t)=T_{x} D_{s} g(t)=s^{-d / 2} g\left(\frac{t-x}{s}\right) .
$$


This representation is square-integrable but reducible. Nevertheless, it has an abundance of admissible vectors $g$ for which the reproducing formula (6) holds. Another way to deal with the reducibility is to study the extended affine group $\mathbb{R}^{d} \times \mathbb{R}_{+} \times S O(d)$ and its representations $\pi_{1}(x, s, R) f(t)=$ $s^{-d / 2} f\left(s^{-1} R^{-1}(t-x)\right)$ with $R \in S O(d)$. Then $\pi_{1}$ is irreducible. For rotationinvariant functions $g$ we have $\pi_{1}(x, s, R) g=\pi(x, s) g$, so we may as well work with the reducible $\pi$. The representation coefficients of $\pi$ are nothing other than the continuous wavelet transform, which is defined by

$$
W_{g} f(x, s)=\langle f, \pi(x, s) g\rangle=s^{-d / 2} \int_{\mathbb{R}^{d}} f(t) \overline{\left(\frac{t-x}{s}\right)} d t
$$

for $f, g \in L^{2}\left(\mathbb{R}^{d}\right), g \neq 0$.

We first identify the coorbit spaces with respect to the representation $\pi$ of $\mathcal{G}_{A}$. Let $1 \leq p, q<\infty$ and $w(x, s)=s^{-\sigma}$ for $\sigma \in \mathbb{R}$. The mixed norm space $L_{\sigma}^{p, q}\left(\mathcal{G}_{\mathrm{A}}\right)$ is defined by the norm

$$
\left\|F \mid L_{\sigma}^{p, q}\left(\mathcal{G}_{\mathrm{A}}\right)\right\|=\left(\int_{0}^{\infty}\left(\int_{\mathbb{R}^{d}}|F(x, s)|^{p} d x\right)^{q / p} s^{-\sigma q} \frac{d s}{s^{d+1}}\right)^{1 / q}
$$

with the usual modifications when $p=\infty$ or $q=\infty$.

Recall the classical definition of the homogeneous Besov spaces. Let $\varphi \in$ $\mathcal{S}\left(\mathbb{R}^{d}\right)$ with $\operatorname{supp}(\varphi) \subset\left\{y \in \mathbb{R}^{d}:|y|<2\right\}$ and $\varphi(x)=1$ if $|x| \leq 1$ and set $\varphi_{j}(x)=\varphi\left(2^{-j} x\right)-\varphi\left(2^{-j+1} x\right), j \in \mathbb{Z}$. For $1 \leq p, q \leq \infty$ and $\sigma \in \mathbb{R}$, the homogeneous Besov space $\dot{B}_{p q}^{\sigma}\left(\mathbb{R}^{d}\right)$ is the set of all tempered distribution modulo polynomials $f \in \mathcal{S}^{\prime} / \mathcal{P}\left(\mathbb{R}^{d}\right)$ such that

$$
\left\|f \mid \dot{B}_{p q}^{\sigma}\left(\mathbb{R}^{d}\right)\right\|=\left(\sum_{j \in \mathbb{Z}} 2^{j \sigma q}\left\|\mathcal{F}^{-1}\left(\varphi_{j} \widehat{f}\right) \mid L^{p}\left(\mathbb{R}^{d}\right)\right\|^{q}\right)^{1 / q}
$$

is finite, with the usual modification for $q=\infty$. A result of Triebel [29] yields the equivalent norm on $\dot{B}_{p q}^{\sigma}\left(\mathbb{R}^{d}\right)$ :

$$
\left(\int_{0}^{\infty} s^{-q(\sigma+d / 2-d / q)}\left\|W_{g} f(\cdot, s) \mid L^{p}\left(\mathbb{R}^{d}\right)\right\|^{q} \frac{d s}{s^{d+1}}\right)^{1 / q}=\left\|W_{g} f \mid L_{\sigma+d / 2-d / q}^{p, q}\left(\mathcal{G}_{\mathrm{A}}\right)\right\| .
$$

Triebel's result reveals that the homogeneous Besov spaces coincide with some coorbit spaces of the affine group $\mathcal{G}_{A}$. More precisely,

$$
\dot{B}_{p q}^{\sigma}\left(\mathbb{R}^{d}\right)=\operatorname{Co}\left(L_{\sigma+d / 2-d / q}^{p, q}\left(\mathcal{G}_{\mathrm{A}}\right)\right) .
$$

Next we compare classical molecules as in [11] and the coorbit molecules according to Definition 3.1. Let us start by describing the classical molecules. For $k=\left(k_{1}, \ldots, k_{d}\right) \in \mathbb{Z}^{d}$ and $j \in \mathbb{Z}$, a dyadic cube is given by $Q=Q_{j k}=$ $\left\{\left(x_{1}, \ldots, x_{d}\right) \in \mathbb{R}^{d}: k_{i} \leq 2^{j} x_{i}<k_{i}+1\right\}$. Its left corner is $x_{Q}=x_{Q_{j k}}=2^{-j} k$, its side length $\ell(Q)=\ell\left(Q_{j k}\right)=2^{-j}$, and its volume is $|Q|=2^{-j d}$. For 
$M, N \in\{-1,0,1,2, \ldots\}$ a classical smooth $(M, N)$-molecule associated to a dyadic cube $Q$ is a function $m_{Q}$ satisfying the estimates

$$
\left|\mathrm{D}^{\alpha} m_{Q}(x)\right| \leq|Q|^{-1 / 2-|\alpha| / d}\left\{1+\frac{\left|x-x_{Q}\right|}{\ell(Q)}\right\}^{-M} \quad \text { for }|\alpha| \leq M, x \in \mathbb{R}^{d},
$$

and the moment conditions

$$
\int_{\mathbb{R}^{d}} x^{\beta} m_{Q}(x) d x=0 \quad \text { for }|\beta| \leq N .
$$

This notion of a classical smooth molecule goes back to [9]; see also $[8,10,11]$. [The atoms in classical analysis are defined similarly with the decay condition (16) replaced by an appropriate support condition.] To understand how the conditions (16) and (17) can be expressed by the wavelet transform, we note that the decay condition (16) can be rephrased as

$$
\left|\mathrm{D}^{\alpha} m_{Q_{j k}}(x)\right| \leq 2^{j d / 2+j|\alpha|}\left(1+\left|2^{j} x-k\right|\right)^{-M} \quad \text { for }|\alpha| \leq M
$$

and the moment conditions (17) as

$$
\widehat{m}_{Q_{j k}}(\xi) \leq C_{n}|\xi|^{n} \quad|\xi| \rightarrow 0 \text {, for all } n \leq N,
$$

The next proposition describes the decay of wavelet transform of the classical molecules.

Proposition 4.4. Let $g$ and $f$ satisfy the conditions (18) with $j=k=0$ and (17). Then there are numbers $\alpha, \beta, \gamma \in \mathbb{N}$ depending only on $M, N$ and a constant $C_{\alpha, \beta, \gamma}>0$ such that

$$
\left|W_{g} f(x, s)\right| \leq C_{\alpha, \beta, \gamma} s^{\alpha}(1+s)^{-\beta}(1+|x|)^{-\gamma} .
$$

By improving the quality of the window we can achieve a stronger result.

REMARK 4.5. In particular, if $g \in \mathcal{S}\left(\mathbb{R}^{d}\right)$ has all moments vanishing, then for every $\alpha, \beta, \gamma \in \mathbb{N}$ there is a constant $C_{\alpha, \beta, \gamma}>0$ such that

$$
\left|W_{g} g(x, s)\right| \leq C_{\alpha, \beta, \gamma} s^{\alpha}(1+s)^{-\beta}(1+|x|)^{-\gamma} .
$$

Proposition 4.4 is due to Holschneider [22], though the result is somewhat hidden in the proofs of his Theorems 11.0.2, 12.0.1, and 19.0.1. ([22] uses a different normalization of the wavelet transform and treats the dimensions $d=1$ and $d>1$ separately.)

The next proposition clarifies the relation between classical molecules and coorbit molecules for the affine group.

Proposition 4.6. Fix a weight function $w$ on $\mathcal{G}_{A}$. Then for $M, N$ sufficiently large, every set of $(M, N)$-molecules $\left(m_{Q_{j k}}\right)$ is a set of coorbit molecules in the sense of Definition 3.1.

Proof. Note that the dyadic cube $Q_{j k}=2^{-j}\left(k+[0,1]^{d}\right)$ is attached to the point $x_{j k}=\left(2^{-j} k, 2^{-j}\right) \in \mathcal{G}_{\mathrm{A}}$. To show that $\left(m_{Q_{j k}}\right)$ is a set of coorbit 
molecules with envelope function $H$, we need to show that

$$
\left|W_{g} m_{Q_{j k}}(x, s)\right| \leq H\left(\left(2^{-j} k, 2^{-j}\right)^{-1}(x, s)\right)=H\left(2^{j} x-k, 2^{j} s\right) .
$$

In view of estimate (19), the natural candidate for an envelope $H$ is the function

$$
H(x, s)=s^{\alpha}(1+s)^{-\beta}(1+|x|)^{-\gamma}
$$

with $\alpha, \beta, \gamma \in \mathbb{N}$ depending on $M, N$. Our task is to show that $H \in$ $W^{R}\left(L^{\infty}, L_{w}^{1}\right)$. We must first estimate the local maximum function $F_{\sharp}^{R}$ of $H$. We set $U=B(0, a) \times\left[b^{-1}, b\right]$ with $a>0$ and $b>1$. Then

$$
\begin{aligned}
F_{\sharp}^{R}(x, s) & =\sup _{(u, v) \in U^{-1}(x, s)^{-1}}|H(u, v)|=\sup _{(y, r) \in U} H\left((y, r)^{-1}(x, s)^{-1}\right) \\
& =\sup _{(y, r) \in U}\left|H\left(-\frac{x+s y}{s r}, \frac{1}{s r}\right)\right| \\
& =\sup _{(y, r) \in U}\left(\frac{1}{s r}\right)^{\alpha}\left(1+\frac{1}{s r}\right)^{-\beta}\left(1+\frac{|x+s y|}{s r}\right)^{-\gamma} \\
& =\sup _{y \in B(0, a)} \sup _{r \in\left[b^{-1}, b\right]}(s r)^{-\alpha+\beta}(1+s r)^{-\beta}(1+|x / s r+y / r|)^{-\gamma} \\
& \leq C_{b} \sup _{y \in B(0, a)} s^{-\alpha+\beta}(1+s)^{-\beta}(1+|x / s+y|)^{-\gamma} \\
& \leq C_{a b} s^{-\alpha+\beta}(1+s)^{-\beta}(1+|x / s|)^{-\gamma} .
\end{aligned}
$$

In the last estimate the moderateness of the weight $(1+|\cdot|)^{-\gamma}$ has been used.

The $W^{R}\left(L^{\infty}, L_{w}^{1}\right)$-norm of $H$ is then

$$
\begin{aligned}
\left\|H \mid W^{R}\left(L^{\infty}, L_{w}^{1}\right)\right\| & =\int_{\mathbb{R}^{d}} \int_{0}^{\infty} F_{\sharp}^{R}(x, s) s^{-\sigma} d x \frac{d s}{s^{d+1}} \\
& \leq C_{a b} \int_{0}^{\infty} \int_{\mathbb{R}} s^{-\alpha+\beta}(1+s)^{-\beta}(1+|x / s|)^{-\gamma} s^{-\sigma} d x \frac{d s}{s^{d+1}},
\end{aligned}
$$

and this integral converges if $\gamma>d$ and $\beta>\alpha+\sigma>0$.

Finally, we apply Theorem 3.5 to study the boundedness of the Hilbert transform on homogeneous Besov spaces. Recall that the Hilbert transform $\mathbf{H}$ of a function $f$ is given by

$$
\mathbf{H} f(x)=\lim _{\varepsilon \rightarrow 0} \frac{1}{\pi} \int_{|t| \geq \varepsilon} \frac{f(x-t)}{t} d t,
$$

provided that the limit exists. The boundedness of $\mathbf{H}$ on Besov spaces follows from Fourier multiplier theorems for Besov spaces (see, e.g., [28]), or from 
Lemarié's work on Calderón-Zygmund operators on Besov spaces [23]. Here we show that it is an immediate consequence of Theorem 3.5.

Proposition 4.7. Let $1 \leq p, q \leq \infty$ and $\sigma \in \mathbb{R}$. Then the Hilbert transform is bounded on $\dot{B}_{p q}^{\sigma}\left(\mathbb{R}^{d}\right)$.

Proof. We choose a basis function $g \in \mathcal{S}(\mathbb{R})$ such that supp $\hat{g} \subseteq$ $\{\omega \in \mathbb{R}: 1 / 2 \leq|\omega| \leq 2\}$ and $\left\{\pi\left(2^{-j} k, 2^{-j}\right) g: j \in \mathbb{Z}, k \in \mathbb{Z}^{d}\right\}$ is a Banach frame for $\dot{B}_{p q}^{\sigma}\left(\mathbb{R}^{d}\right)$. Since the Hilbert transform commutes with all translations $T_{x}$ and dilations $D_{s}$, i.e., $\mathbf{H}\left(T_{x} D_{s}\right) f(t)=T_{x} D_{s}(\mathbf{H} f)(t) \mathbf{H}$ maps the frame $\pi\left(2^{-j} k, 2^{-j}\right) g$ into atoms $\pi\left(2^{-j} k, 2^{-j}\right) \mathbf{H} g$. Therefore it suffices to prove that $W_{g} \mathbf{H} g \in W^{R}\left(L^{\infty}, L_{w}^{1}\right)\left(\mathcal{G}_{A}\right)$ where $w(x, s)=s^{-\sigma+d / 2-d / q}$; then $K=\left|W_{g} \mathbf{H} g\right|$ serves as an envelope for which (8) holds and $\mathbf{H}\left(\pi\left(2^{-j} k, 2^{-j}\right)\right) g$, $j, k \in \mathbb{Z}$, is a set of molecules. Since both $g$ and $\mathbf{H} g$ are in $\mathcal{S}$ with all moments vanishing, estimate (19) and the proof of Proposition 4.6 show that $W_{g} \mathbf{H} g \in W^{R}\left(L^{\infty}, L_{w}^{1}\right)\left(\mathcal{G}_{A}\right)$.

Acknowledgments. Both authors were supported by the Marie-Curie Excellence Grant MEXT-CT 2004-517154.

\section{References}

[1] R. M. Balan, P. G. Casazza, C. Heil, and Z. Landau, Density, overcompleteness, and localization of frames II: Gabor frames, J. Fourier Anal. Appl. 12 (2006), 307-344.

[2] R. R. Coifman, A real variable characterization of $H^{p}$, Studia Math. 51 (1974), 269-274.

[3] R. R. Coifman, R. Rochberg, H. M. Taibleson, and G. Weiss, Introduction, in: Representation Theorems for Hardy Spaces, Astérisque 77 (1980), 1-9.

[4] S. Dahlke, G. Kutyniok, G. Steidl, and G. Teschke, Shearlet coorbit spaces and associated Banach frames, preprint, 2007.

[5] H. G. Feichtinger and K. Gröchenig, A unified approach to atomic decompositions via integrable group representations, in: Function, Spaces and Applications (Lund, 1986), Lecture Notes in Math. 1302, Springer, 1988, 52-73.

[6] —, - Banach spaces related to integrable group representations and their atomic decompositions, I, J. Funct. Anal. 86 (1989), 307-340.

[7] - - - Banach spaces related to integrable group representations and their atomic decompositions, II, Monatsh. Math. 108 (1989), 129-148.

[8] M. Frazier, Y.-S. Han, B. Jawerth, and G. Weiss, The T1 theorem for TriebelLizorkin spaces, in: Harmonic Analysis and Partial Differential Equations (El Escorial, 1987), Lecture Notes in Math. 1384, Springer, Berlin, 1989, 168-181.

[9] M. Frazier and B. Jawerth, Decomposition of Besov spaces, Indiana Univ. Math. J. 34 (1985), 777-799.

[10] - - - A discrete transform and decompositions of distribution spaces, J. Funct. Anal. 93 (1990), 34-170.

[11] M. Frazier, B. Jawerth and G. Weiss, Littlewood-Paley Theory and the Study of Function Spaces, CBMS Reg. Conf. Ser. Math. 79, Amer. Math. Soc., Providence, RI, 1991. 
[12] H. Führ, Abstract Harmonic Analysis of Continuous Wavelet Transforms, Lecture Notes in Math. 1863, Springer, Berlin, 2005.

[13] L. Grafakos, Classical and Modern Fourier Analysis, Pearson Education, Upper Saddle River, NJ, 2004.

[14] L. Grafakos and R. Torres, Pseudodifferential operators with homogeneous symbols, Michigan Math. J. 46 (1999), 261-269.

[15] K. Gröchenig, Describing functions: atomic decompositions versus frames, Monatsh. Math. 112 (1991), 1-41.

[16] -, Foundations of Time-Frequency Analysis, Appl. Numer. Harmonic Anal., Birkhäuser Boston, Boston, MA, 2001.

[17] - Localization of frames, Banach frames, and the invertibility of the frame operator, J. Fourier Anal. Appl. 10 (2004), 105-132.

[18] -, Time-Frequency Analysis of Sjöstrand's class, Rev. Mat. Iberoamer. 22 (2006), 703-724.

[19] K. Gröchenig, E. Kaniuth, and K. F. Taylor, Compact open sets in duals and projections in $L^{1}$-algebras of certain semi-direct product groups, Math. Proc. Cambridge Philos. Soc. 111 (1992), 545-556.

[20] K. Gröchenig and Z. Rzeszotnik, Banach algebras of pseudodifferential operators and their almost diagonalization, Ann. Inst. Fourier (Grenoble) 58 (2008), 2279-2314.

[21] A. Grossmann, J. Morlet, and T. Paul, Transforms associated to square integrable group representations. I. General results, J. Math. Phys. 26 (1985), 2473-2479.

[22] M. Holschneider, Wavelets, An Analysis Tool, Oxford Univ. Press, 1995.

[23] P. G. Lemarié, Continuité sur les espaces de Besov des opérateurs définis par des intégrales singulières, Ann. Inst. Fourier (Grenoble) 35 (1985), 175-187.

[24] H. Rauhut, Coorbit space theory for quasi-Banach spaces, Studia Math. 180 (2007), 237-253.

[25] E. M. Stein, Harmonic Analysis: Real-Variable Methods, Orthogonality, and Oscillatory Integrals, Princeton Univ. Press, Princeton, NJ, 1993.

[26] J. Toft, Continuity properties for modulation spaces, with applications to pseudodifferential calculus. II, Ann. Global Anal. Geom. 26 (2004), 73-106.

[27] R. H. Torres, Boundedness results for operators with singular kernels on distribution spaces, Mem. Amer. Math. Soc. 90 (1991), no. 442.

[28] H. Triebel, Theory of Function Spaces, Birkhäuser, Basel, 1983.

[29] -, Characterizations of Besov-Hardy-Sobolev spaces: A unified approach, J. Approx. Theory 52 (1988), 162-203.

Faculty of Mathematics

University of Vienna

Nordbergstrasse 15

A-1090 Vienna, Austria

E-mail: karlheinz.groechenig@univie.ac.at

mariusz.piotrowski@univie.ac.at

Received July 31, 2008

Revised version October 15, 2008 